\title{
Robotic-assisted proximal gastrectomy using the double-flap technique for early gastric cancer with situs inversus totalis: a case report
}

\author{
Atsushi Takeno ${ }^{1,2^{*}}$, Toru Masuzawa', Shinsuke Katsuyama ${ }^{1}$, Kohei Murakami ${ }^{3}$, Kenji Kawai ${ }^{1}$, Yoshiteru Katsura ${ }^{1}$, \\ Yoshiaki Ohmura', Yoshinori Kagawa' ${ }^{1}$ Yutaka Takeda' ${ }^{1}$ Taishi Hata ${ }^{1}$ and Kohei Murata ${ }^{1}$
}

\begin{abstract}
Background: The robotic system has been applied in the treatment of gastric cancer (GC), and the procedure has been found to be safe and feasible. Situs inversus totalis (SIT) is a relatively rare autosomal recessive congenital anomaly. We successfully performed robot-assisted proximal gastrectomy (RAPG) and handsewn double-flap esophagogastrostomy for GC in a patient with SIT.

Case presentation: A 71-year-old woman was referred to us with an asymptomatic ulcerative lesion in the upper body of the stomach. Computed tomography revealed that she had SIT. She was diagnosed with cT1 bNOMO, cStagelA gastric cancer. RAPG with lymph node dissection and handsewn double-flap esophagogastrostomy was performed. Robotic surgery enabled the surgeon to perform the surgery without changing his position and experiencing any confusion resulting from the patient's reversed anatomy. It took $448 \mathrm{~min}$, and no intraoperative complications occurred. Her postoperative course was uneventful; she was discharged on postoperative day 10 . The final pathologic report showed pT1b1NOM0, pStage IA.
\end{abstract}

Conclusions: This is the first case describing RAPG with handsewn double-flap esophagogastrostomy for a SIT patient with early GC.

Keywords: Gastrectomy, Robotic surgical procedures, Situs inversus

\section{Background}

Situs inversus totalis (SIT) is a relatively rare autosomal recessive congenital anomaly found in 1 per $4000-8000$ persons. It is characterized by reverse positioning of the major visceral organs from their usual positions. Laparoscopic gastrectomy (LG) in SIT patients with gastric cancer (GC) was first reported in 2003, and standard typical lymph node dissection has been reported recently in $2010[1,2]$.

The first case of robot-assisted gastrectomy (RG) in SIT patients with GC was reported in 2012 [3]. Kim

\footnotetext{
*Correspondence: atakeno1008@me.com

2 Department of Surgery, National Hospital Organization Osaka National

Hospital, 2-1-14, Hoenzaka, Chuoku, Osaka 540-0006, Japan

Full list of author information is available at the end of the article
}

mentioned that RG was more suitable than LG, because the surgeon did not have to change his position and could change hand easily. Five SIT cases with GC who underwent RG have been reported (3 DG and 2 TG cases) in the past [4-7]. However, to the best of our knowledge, there are no reports of robotic-assisted proximal gastrectomy (RAPG) for upper-third GC in the literature.

The double-flap esophagogastrostomy technique (DFT) has been applied to laparoscopic PG to prevent reflex. In spite of effective prevention of reflex, it is technically demanding because of complicated suturing and ligation maneuvers. Shibasaki reported that RG was more advantageous to perform intracorporeal hand-sewing anastomosis than LG, because robotic assistance could allow to master it with a short learning curve [8]. We describe a 
case of RAPG with lymph node dissection and handsewn DFT for upper-third early GC (EGC).

\section{Case presentation}

An asymptomatic ulcerative lesion was found during screening gastroscopy of the upper body of the stomach in a 71-year-old woman. On the endoscopic ultrasonogram, the 2-cm ulcerative lesion was invading the submucosal layer. Computed tomography (CT) revealed that she had SIT without lymph node or distant metastases, and three-dimensional (3D) reconstruction of an abdominal CT angiogram showed no vessel anomalies (Fig. 1).

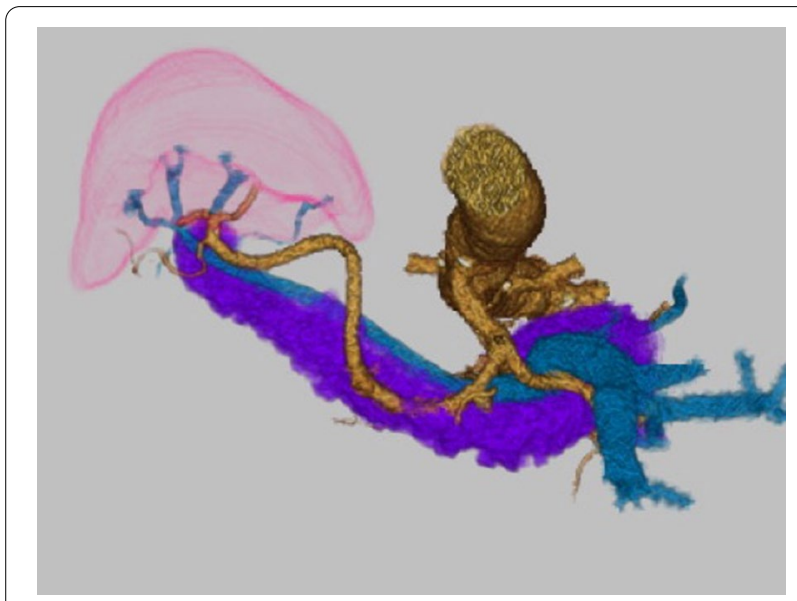

Fig. 1 Three-dimensional reconstruction of an abdominal computed tomography angiogram preoperatively
We diagnosed her with EGC, U, post, cType 0-IIc, cT1bN0M0, cStageIA, and it was thought to be contraindicated for endoscopic resection. We decided to perform robotic-assisted PG (using da Vinci Xi Surgical Systems) and D1+lymphadenectomy based on Japanese gastric cancer treatment guidelines 2018 (5th edition) [9].

A scope was inserted into the abdominal cavity through an 8 - $\mathrm{mm}$ port on the umbilicus, and four more trocars were inserted. As shown in Fig. 2, the location of the trocar placement is the same as usual, whereas we adjusted their arrangement. In performing RAG, we normally set 4th arm at the left lateral side to develop macro-surgical field and handle 1st arm at the right lateral and 3rd arm at the left medial to proceed the surgical procedure. In this SIT case, we adjusted to set 1st arm at the right lateral as developing the field and handle $2 \mathrm{nd}$ at the left medial and 4th at the left medial, because it might be difficult to approach to the esophagus and cardia located in the right upper area if we had adopted normal setting. Accordingly, assistant trocar was moved from the right medial to the left lateral.

The liver was retracted with internal organ retractors. After a thorough examination, the greater omentum was divided $3 \mathrm{~cm}$ away from the gastroepiploic vessels. The right (left in the normal anatomy) gastroepiploic artery and vein were clipped and divided near the spleen. The gastrosplenic ligament was divided by a sealing device. Next, the lesser omentum was opened and the lymph nodes along the lesser curvature of the stomach were dissected. We moved to the suprapancreatic area, and lymph node numbers $8 \mathrm{a}, 9$, and 7 were dissected safely.

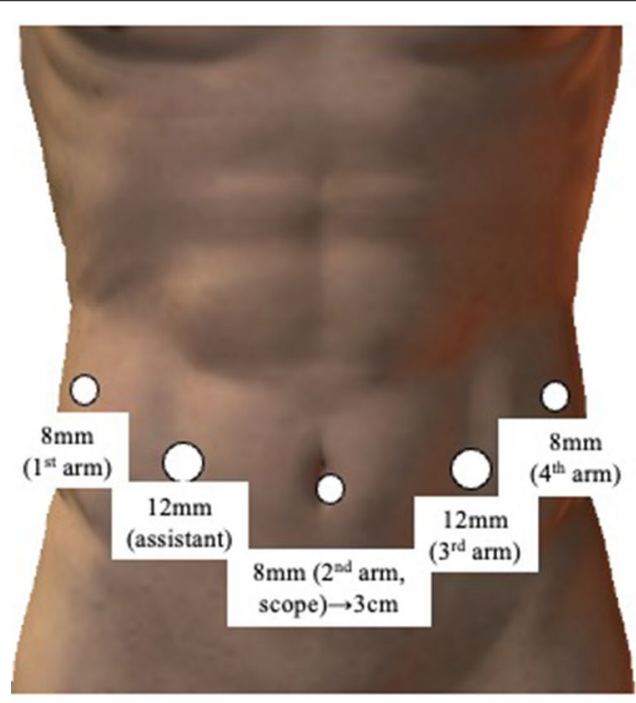

(a) Ordinary trocar placement

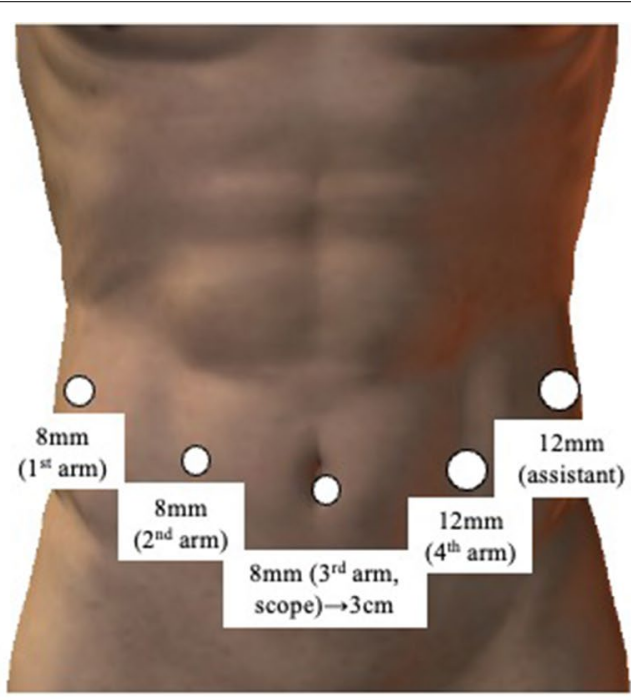

(b) This SIT case

Fig. 2 Placement of the trocars and robotic arms. a Ordinary trocar placement. b This SIT case 
Finally, by tracing the splenic artery behind the splenic vein, the number 11 group of lymphatic tissue was harvested. The postperitoneal fascia between the upper border of the pancreatic tail and cardia was dissected along a plane superior to the Gerota fascia. The pericardiac and periesophageal tissue in the esophageal hiatus was dissected, and the esophagus was transected with the Endowrist Stapler. The stomach was extracted through a $3-\mathrm{cm}$ umbilical incision and resected at the level of the upper one-third of the stomach.

The double seromuscular flaps were prepared extracorporeally at the anterior wall of the remnant stomach in order to cautiously remove the submucosal layer from the mucosal layer. After establishing the pneumoperitoneum again, we performed handsewn esophagogastrostomy intracorporeally. Firstly, four stitches were used to fix the posterior wall of the esophagus to the superior edge of the mucosal window. Secondly, the posterior wall of the esophagus and the superior opening of the mucosa on the remnant stomach were closed by continuous suturing using barbed sutures. The anterior wall of the esophagus and gastric wall at the lower end of the flap were also anastomosed layer-by-layer by continuous suturing using barbed sutures. Finally, the anastomosis was finished by covering the anastomosis site with seromuscular flaps using barbed sutures. An air leakage test was performed to confirm closure of the anastomosis (Fig. 3).

The operative time was $448 \mathrm{~min}$, and blood loss was $45 \mathrm{ml}$. The final pathology demonstrated a poorly differentiated 0-IIc lesion with invasion to the submucosa. There was no metastasis in any of the retrieved lymph nodes. The final stage was pT1b1N0M0, pStage IA according to the Japanese Classification of Gastric Carcinoma staging system. No intraoperative complications

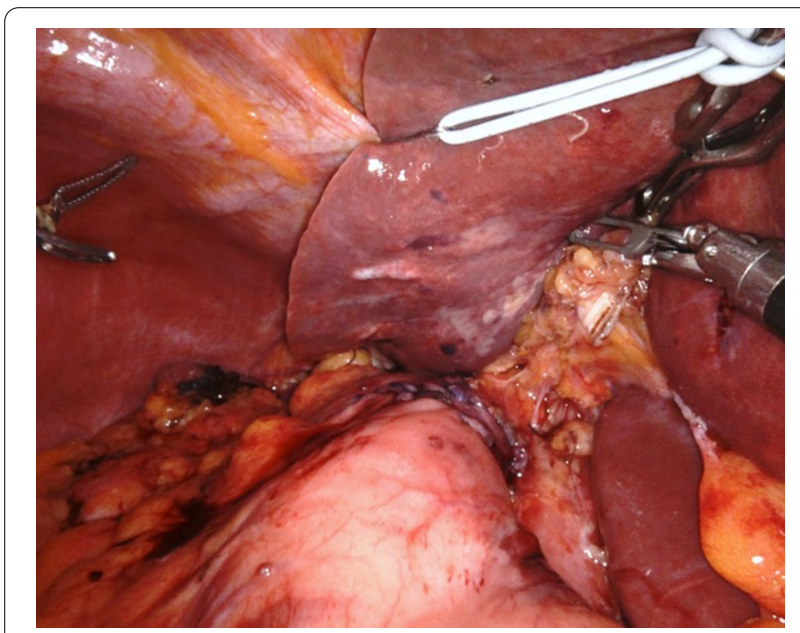

Fig. 3 Intraoperative findings after esophagogastrostomy occurred. The patient's postoperative course was uneventful; she was discharged on postoperative day 10.

\section{Discussion}

SIT is a rare congenital anomaly. SIT can be accompanied by cardiopulmonary malformations, familial long QT syndrome, total esophageal duplication, agnathia, and various urologic anomalies [10]. Therefore, it is important to identify any abnormal vasculature preoperatively, because it is associated with a risk of misidentifying anatomy and causing unanticipated injury of important vessels. Our patient had no major vascular abnormalities. However, it is necessary to examine $3 \mathrm{D}$ reconstruction of a blood stream from an abdominal CT angiogram preoperatively, as shown in Fig. 1.

RG for GC has been developed for minimally invasive surgery and is thought to have the potential to overcome the shortcomings of LG. A comparative study demonstrated that RG is as acceptable as LG in surgical and oncologic outcomes with lower estimated blood loss, acceptable complications, and radical resection [11]. A recent study about long-term oncologic outcomes of RG and LG revealed that RG is an oncological alternative [12].

Only small cases of GC have been reported in patients with SIT to date. Of these, only five patients underwent RG, 3 for DG $[3,5,7]$ and the other 2 for TG $[4,6]$. Additionally, nine patients underwent laparoscopic-assisted DG and only one patient underwent TG [10]. In laparoscopic surgery, little ingenuity in moving the monitor and standing opposite the usual side are required. However, in robotic surgery like in our case, the surgeon could perform the operation without changing his position and experiencing any confusion resulting from the patient's reversed anatomy. Indeed, we successfully completed RAPG in the same procedure as normal cases besides adjusting the trocar arrangement.

We adopted the double-flap esophagogastrostomy technique (DFT) after PG. This technique was developed to prevent reflux after PG has been applied to laparoscopic proximal gastrectomy (LPG-DFT) [13]. It is achieved by burying the abdominal esophagus into the gastric submucosa. It has been reported to be a better surgical procedure for treating upper-third EGC than laparoscopic TG in morbidity, postoperative hospital stay, and postoperative nutritional status [14]. Kuroda also reported that it was a feasible option for effective prevention of reflex based on the results of multicenter retrospective study [15]. However, LPG-DFT is technically demanding because of complicated suturing and ligation maneuvers. Robotic assistance may be useful for valvuloplastic esophagogastrostomy-DFT with a short learning curve [8]. 


\section{Conclusion}

This is the first case describing RAPG with handsewn double-flap esophagogastrostomy for a SIT patient with early GC. RG may be suitable to perform proximal gastrectomy for such a complicated SIT case because it can resolve technical problems.

\section{Acknowledgements}

We would like to thank Editage (www.editage.com) for English language editing.

\section{Authors' contributions}

AT, TM, and KM managed the patient. AT wrote the manuscript and provided the original pictures. All the other authors reviewed the manuscript. All authors approved the content of the manuscript. All authors read and approved the final manuscript.

\section{Funding}

The authors have no funding regarding this paper.

\section{Availability of data and materials}

Data sharing is not applicable to this article as no datasets were generated or analyzed during the current study.

\section{Declarations}

\section{Ethics approval and consent to participate}

This case report was approved by a constituted ethics committee of our hospital, and it conforms to the provisions of the 1995 Declaration of Helsinki (as revised in Brazil 2013).

\section{Consent for publication}

Written informed consent was obtained from the patient for publication of this case report and any accompanying images. A copy of the written consent is available for review by the Editor-in-Chief of this journal.

\section{Competing interests}

The authors declare that they have no competing interests.

\section{Author details}

${ }^{1}$ Department of Gastrointestinal Surgery, Kansai Rosai Hospital, Amagasaki, Japan. ${ }^{2}$ Department of Surgery, National Hospital Organization Osaka National Hospital, 2-1-14, Hoenzaka, Chuoku, Osaka 540-0006, Japan. ${ }^{3}$ Department of Surgery, Japan Community Heath Care Organization Osaka Hospital, Osaka, Japan.

Received: 21 June 2021 Accepted: 26 July 2021

Published online: 02 August 2021

\section{References}

1. Yamaguchi S, Orita H, Yamaoka T, Mii S, Sakata H, Hashizume M. Laparoscope-assisted distal gastrectomy for early gastric cancer in a 76-year-old man with situs inversus totalis. Surg Endosc. 2003;17:352-3.
2. Futawatari N, Kikuchi S, Moriya H, Katada N, Sakuramoto S, Watanabe M. Laparoscopy-assisted distal gastrectomy for early gastric cancer with complete situs inversus: report of a case. Surg Today. 2010;40:64-7.

3. Kim HB, Lee JH, Park DJ, Lee HJ, Kim HH, Yang HK. Robot-assisted distal gastrectomy for gastric cancer in a situs inversus totalis: a case report. J Korean Surg Soc. 2012;82:321-4.

4. Cao Y, Li J, Shen L, Wang J, Xia Z, Tao K, et al. Gastric cancer in a situs inversus totalis patient with multiple intestinal and vessel variations related to gastrectomy surgery: a case report and literature review. Medicine (Baltimore). 2017;96:8209.

5. Alhossaini R, Hyung WJ. Robotic assisted distal gastrectomy for gastric cancer in a patient with situs inversus totalis: with Video. J Gastrointest Surg. 2017;21(12):2144-5.

6. Yoshimoto T, Yoshikawa K, Tokunaga T, Nishi M, Takasu C, Kashihara H, et al. Robotic-assisted total gastrectomy in a patient with gastric cancer associated with situs inversus totalis: with video. Asian J Endosc Surg. 2021;14(2):297-300

7. Abbey E, Yang F, Qi L, Wu J, Tong L, Zhen Z. Situs inversus totalis patients with gastric cancer: robotic surgery the standard of treatment? A case report. Int J Surg Case Rep. 2021:81:105818.

8. Shibasaki S, Suda K, Nakauchi M, Kikuchi K, Kadoya S, Ishida Y, et al. Robotic valvuloplastic esophagogastrostomy using double flap technique following proximal gastrectomy: technical aspects and short-term outcomes. Surg Endosc. 2017;31:4283-97.

9. Japanese Gastric Cancer Association. Japanese gastric cancer treatment guidelines 2018 (5th edition). Gastric cancer 2021; 24: 1-21.

10. Morimoto M, Hayakawa T, Kitagami H, Tanaka M, Matsuo Y, Takeyama H. Laparoscopic-assisted total gastrectomy for early gastric cancer with situs inversus totalis: report of a first case. BMC Surg. 2015;15:75.

11. Shen W, Xi H, Wei B, Cui J, Bian S, Zhang K, et al. Robotic versus laparoscopic gastrectomy for gastric cancer: comparison of short-term surgical outcomes. Surg Endosc. 2016;30:574-80.

12. Obama K, Kim YM, Kang DR, Son T, Kim H, Noh SH, et al. Long-term oncologic outcomes of robotic gastrectomy for gastric cancer compared with laparoscopic gastrectomy. Gastric Cancer. 2018;21:285-95.

13. Kuroda S, Nishizaki M, Kikuchi S, Noma K, Tanabe S, Kagawa S, et al. Double-flap technique as an antireflux procedure in esophagogastrostomy after proximal gastrectomy. J Am Coll Surg. 2016;223:e7-13.

14. Hayami M, Hiki N, Nunobe S, Mine S, Ohashi M, Kumagai K, et al. Clinical outcomes and evaluation of laparoscopic proximal gastrectomy with double-flap technique for early gastric cancer in the upper third of the stomach. Ann Surg Oncol. 2017;24:1635-42.

15. Kuroda S, Choda Y, Otsuka S, Ueyama S, Tanaka N, Muraoka A, et al. Multicenter retrospective study to evaluate the efficacy and safety of the double-flap technique as antireflux esophagogastrostomy after proximal gastrectomy (rD-FLAP Study). Ann Gastroenterol Surg. 2019;3(1):96-103.

\section{Publisher's Note}

Springer Nature remains neutral with regard to jurisdictional claims in published maps and institutional affiliations.

\section{Submit your manuscript to a SpringerOpen ${ }^{\circ}$ journal and benefit from:}

- Convenient online submission

$\checkmark$ Rigorous peer review

- Open access: articles freely available online

- High visibility within the field

- Retaining the copyright to your article

Submit your next manuscript at $\boldsymbol{\nabla}$ springeropen.com 